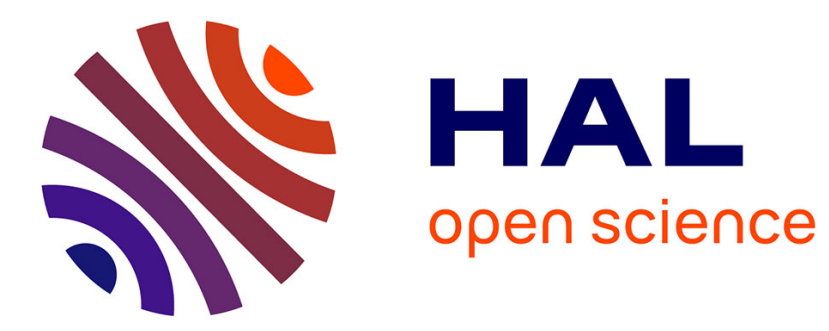

\title{
Quelques données relatives à l'historiographie lao
}

Michel Lorrillard

\section{To cite this version:}

Michel Lorrillard. Quelques données relatives à l'historiographie lao. Bulletin de l'Ecole française d'Extrême-Orient, 1999, 86, pp.219-232. 10.3406/befeo.1999.3412 . hal-02358492

\section{HAL Id: hal-02358492 \\ https://hal.science/hal-02358492}

Submitted on 12 Nov 2019

HAL is a multi-disciplinary open access archive for the deposit and dissemination of scientific research documents, whether they are published or not. The documents may come from teaching and research institutions in France or abroad, or from public or private research centers.
L'archive ouverte pluridisciplinaire HAL, est destinée au dépôt et à la diffusion de documents scientifiques de niveau recherche, publiés ou non, émanant des établissements d'enseignement et de recherche français ou étrangers, des laboratoires publics ou privés. 


\section{Michel Lorrillard}

\section{Quelques données relatives à l'historiographie lao}

In: Bulletin de l'Ecole française d'Extrême-Orient. Tome 86, 1999. pp. 219-232.

Citer ce document / Cite this document :

Lorrillard Michel. Quelques données relatives à l'historiographie lao. In: Bulletin de l'Ecole française d'Extrême-Orient. Tome 86, 1999. pp. 219-232.

doi : $10.3406 /$ befeo. 1999.3412

http://www.persee.fr/web/revues/home/prescript/article/befeo_0336-1519_1999_num_86_1_3412 


\title{
Quelques données relatives à l'historiographie lao
}

\author{
Michel Lorrillard *
}

Durant les années 1950-1970, sous l'égide de deux institutions successives, le Comité littéraire et l'Académie royale du Laos, un certain nombre de lettrés lao ont commencé à rédiger des ouvrages présentant d'une façon synthétique l'histoire de leur pays. Le plus important d'entre eux fut sans aucun doute le Maha Sila Viravong, personnage dont la notoriété fut telle qu'une biographie ${ }^{1}$ lui a été récemment consacrée, privilège jusqu'ici encore rare au Laos. Si le champ d'érudition du Maha Sila Viravong fut large et divers - il couvrit également les domaines de la littérature, de la grammaire et de la linguistique -, c'est principalement l'œuvre de l'historien que l'on a retenue et qui prévaut encore. L'ouvrage intitulé Phongsāvadān Lao (Histoire du Laos), édité une première fois en 1957 puis repris intégralement pour servir de texte aux manuels d'histoire de l'enseignement secondaire $^{2}$, connut une diffusion qui dépassa les frontières nationales puisqu'il fut traduit en anglais ${ }^{3}$ et sert encore aujourd'hui de base à la plupart des travaux anglo-saxons sur l'histoire ancienne du Laos. Il est rédigé d'une manière linéaire (malgré un découpage en chapitres) et donne l'impression d'un ensemble homogène, comme si le sujet pouvait être traité dans sa globalité sans difficultés. En fait, le texte emprunte à des sources diverses que l'auteur ne cite pratiquement jamais. Sur le plan formel, le travail du Maha Sila Viravong ne se distingue d'ailleurs guère des chroniques auxquelles l'on pourra avoir accès par ailleurs. Il apparaît simplement plus complet, plus lisible et apparemment plus logique. Il s'agit en fait d'un vaste travail de compilation et de polissage des sources, dont nous imaginons volontiers qu'il a nécessité un certain nombre de choix et d'interprétations, sans que les critères ayant guidé ceux-ci apparaissent jamais d'une façon explicite.

Toutefois, si l'on se reporte à la préface que le Maha Sila Viravong donne à son ouvrage à l'intention des lycées, on trouve, à partir d'un exemple donné concernant le personnage de Fā Ngum, premier souverain historique du Lān Xāng, un exposé très net des considérations qui orientèrent le lettré dans la conception de son ouvrage. On voit que si celui-ci accorde d'une façon générale un crédit analogue à chacune des chroniques dont il se sert, il exerce par contre une sorte de censure pour certaines parties d'entre elles, traitant de faits précis, sous prétexte qu'elles contiennent des éléments tout à fait incompatibles avec sa conception de la vérité historique. Le fait apparaît d'autant plus surprenant lorsque l'on considère les éléments incriminés. Il s'agit en l'occurrence des

* Membre de l'École française d'Extrême-Orient.

1. Maha Sila Viravong, Sivit Lè Phon Ngan [Maha Sila Viravong, la vie et l'œuvre], 1990. Voir références bibliographiques infra.

2. Maha Sila Viravong, Phongsāvadān Lao, 1957.

3. Maha Sila Viravong, History of Laos, 1958. 
raisons ayant entraîné l'exil de Fā Ngum (vers 1316) et des conditions ayant entouré l'accès au trône de ce dernier. Il existe, à propos de ces événements, deux grandes traditions dans les chroniques lao. La première est celle du Nithān Khun Borom, texte dont la première rédaction date très certainement du début du $X I^{e}$ siècle. Fā Ngum y est présenté comme le fils d'un certain Fā Ngieo (ou Kham Hieo suivant les versions). Comme il était "sorti du ventre de sa mère » avec trente-trois dents, les devins de Xieng Dong Xieng Thong (Luang Prabang ${ }^{4}$ ) jugèrent que le présage était mauvais et l'enfant fut exilé, accompagné seulement d'un nombre restreint de serviteurs. Le radeau sur lequel on les avait placés finit par arriver à Nakhon Luang (Angkor) où Fā Ngum resta jusqu'à l'annonce de la mort de ses parents. Avec l'aide du souverain khmer - qui l'avait recueilli et lui avait donné sa fille en mariage - il constitua alors une armée et conquit petit à petit ce qui allait devenir le grand territoire du Lān Xāng, y compris le domaine de Luang Prabang où il rentrait sans trop de difficultés pour s'y faire couronner roi (1353). La seconde tradition est celle des phongsāvadān, famille de textes souvent recopiés et modifiés, mais dont l'origine est probablement au moins aussi ancienne que celle du Nithān Khun Borom (Lorrillard, 1995 : 319). On y rapporte que Fā Ngum est le fils d'un certain Phī Fā, lequel aurait eu une relation incestueuse et adultère avec l'une des femmes de son père - le roi de Luang Prabang -, ce qui lui aurait valu d'être exilé avec son fils. Tous deux aboutirent au pays khmer où ils restèrent de nombreuses années, puis, l'occasion étant favorable, ils revinrent vers Luang Prabang, conquérant au passage nombre de territoires. À Xieng Khuang, où ils s'étaient arrêtés, il fut décidé que Fā Ngum prendrait la route la plus courte en suivant le cours de la Nam Khān pendant que son père ferait un détour pour rattraper les cours de la Nam Xeng et de la Nam Xuang. Fā Ngum arriva le premier à Luang Prabang. Certaines versions rapportent alors que, bien décidé à gouverner seul, il aurait envoyé des tueurs qui le débarrassèrent de son père. Selon d'autres versions, Phī Fà serait mort d'une maladie avant même de parvenir à la capitale, laissant ainsi son fils seul maître du pouvoir.

Le Maha Sila Viravong ne fait état dans son Phongsāvadān Lao que de la seule version où Fā Ngum est exilé avec ses serviteurs. La raison en est expliquée dans cette fameuse préface qu'il donne à l'édition faite à l'intention des lycées. L'auteur s'y interroge sur le fait que la tradition évoquant le personnage de Phī Fā soit si peu représentée ${ }^{5}$ et surtout considère qu'un texte faisant état d'un meurtre perpétré par Fã Ngum sur la personne de son père ne saurait être acceptable. "Cet élément [du récit] - rapporte-t-il excède tout à fait l'entendement dans la mesure où Khun Phī Fā et Thāo Fā Ngum, le père et le fils, ont vécu les mêmes difficultés et ont souffert ensemble : se pourrait-il donc que Fā Ngum ait été méchant et ingrat [à l'égard de son père] ? ». Assurément non, répond indirectement le lettré qui, d'une façon que l'on pourra juger quelque peu partiale, essaie de redorer l'image du prince. Et de rajouter que, dans ces conditions, tout porte à croire que le père de Fā Ngum ne fut jamais exilé et qu'il régna à Luang Prabang ainsi que le rapporte la tradition initiale du Nithān Khun Borom (Viravong, 1973).

Au-delà des raisonnements elliptiques du Maha Sila Viravong, on retient surtout de cet exemple l'énorme pression que des facteurs culturels peuvent exercer sur l'esprit critique de l'historien lao. Contrairement à l'esprit moderne occidental qui aura tendance, en premier lieu, à privilégier le rationnel par rapport à l'irrationnel, c'est ici exactement

4. Pour le nom de la ville, nous donnons ici l'orthographe la plus usitée. Nous choisirons cependant la notation plus correcte de «Phra Bāng » (séparation des deux termes, restitution de l'aspirée et de la voyelle longue) dès lors qu'il s'agit de la célèbre statue.

5. Il s'avère au contraire que le nombre de versions des phongsāvadān (et donc de textes citant Phī Fā) est largement supérieur à celui des versions du Nithān Khun Borom. 
l'inverse qui se produit. Le critère est autre : il est d'abord moral. À une version où sont évoqués des faits réprouvables mais rendus possibles par la nature humaine tels que l'adultère, l'inceste et le parricide, on en préférera une autre où le merveilleux n'est pas rejeté et domine même. L'irrationnel est au Laos, comme dans bien d'autres pays voisins, une donnée parfaitement intégrée, reconnue, valorisée et qui n'entraîne, dans la conscience collective, aucun blocage. Mieux, on se gardera bien, consciemment ou non, d'analyser la nature et le pourquoi des éléments faisant la part au merveilleux. Ceux-ci sont partie intégrante des chroniques et constituent d'ailleurs, pour une grande part, leur essence même. Reprenons l'exemple des trente-trois dents de Fā Ngum, signe physique emprunté à l'évidence à l'imagerie bouddhique ${ }^{6}$. Toucher à ce signe, c'est amputer d'une façon certaine l'image exceptionnelle du souverain et donc en quelque sorte contester sa légitimité. L'historien lao moderne ${ }^{7}$, dans son œuvre de compilation, perpétue une tradition plus qu'il n'en analyse les fondements. Pour mieux saisir ce processus, il sera nécessaire de revenir sur un certain nombre d'aspects propres à l'historiographie ancienne lao.

\section{Les chroniques du Laos : une nécessaire distinction}

$\mathrm{Si}$, par extension, le terme phongsāvadān finit par prendre le sens d' " histoire » ou d' « annales », il évoque à l'origine l'idée de perpétuation d'une lignée ou d'une dynastie ${ }^{8}$. Il est possible que le vocable ait été employé assez tardivement au Laos et qu'il résulte d'un emprunt effectué au vocabulaire siamois. On sait qu'à partir de la fin du XVIII ${ }^{e}$ siècle, les princes lao eurent à se rendre fréquemment auprès de la cour de Bangkok et que l'influence idéologique qu'ils y subirent affecta profondément leur identité culturelle. Pour le $\mathrm{XIX}^{\mathrm{e}}$ siècle, nous avons plusieurs exemples de phongsävadān composés sur ordre du Siam à Luang Prabang et à Champassak (Phinit, 1987: 2 ; Archaimbault, 1961: 579). L'influence siamoise fut telle, pour les plus récents de ces textes, qu'elle apparaît non seulement dans l'introduction de nouveaux termes mais également dans la graphie des caractères et dans l'orthographe de certains mots (Phinit, 1987: 3-4). Le terme phongsāvadān aurait alors pu supplanter celui plus commun de tamnān et confiner ce dernier dans un emploi à la fois plus limité d'un point de vue géographique, et plus large dans son acception. Limité d'abord d'un point de vue géographique car le tamnān a tendance au Laos à recouvrir des histoires locales, celles de cités dont l'autorité politique n'est pas forcément importante mais également celles de lieux voire de bâtiments. Large

6. On fera le parallèle avec les lakșana, terme sanskrit désignant les marques, les attributs ou les caractères physiques du "Grand Homme » (skt mahäpurusa). Les lakṣana sont visibles dès la naissance. Le Bouddha en possédait trente-deux, l'un d'eux étant la possession de quarante dents. Le chiffre trentetrois est important dans la mythologie bouddhique : c'est par exemple le nombre donné aux dieux résidant dans le ciel où renaissent les gens de mérite. Selon certains textes pāli, c'est également le nombre de têtes de l'éléphant Erāvaṇa, la monture d'Indra.

7. Nous prenons comme exemple le Maha Sila Viravong mais nos remarques valent également pour les historiens lao de sa génération : Chao Khamhmanh Vongkot Rattana, Oukham Phomvongsa, Maha Kham Champakeomany, etc. (références en bibliographie). Nous n'aborderons pas ici la question de l'écriture de l'histoire au Laos depuis la révolution communiste de 1975.

8. «Phongsāvadān » est la prononciation thai / lao d'un composé d'origine indienne : Skt vamśa / P. varisa (famille, lignée, dynastie) + Skt avatära (descente). Selon S. Pou (1989: 108), le terme «avatāra » désigne également une personne distinguée ou un prince et «il est plus probable que bañsāvatār ait été composé selon la syntaxe sud-est asiatique, en khmer ou en siamois (déterminédéterminant) : "la lignée (banisā) des princes (avatār)" ". 
ensuite dans son acception puisque le vocable désigne aussi des chroniques qui s'apparentent aux textes hagiographiques : il y sera alors question d'images précieuses du Bouddha, d'histoire de saints ou - la chose étant souvent liée - de monuments révérés et de sites sacrés ${ }^{9}$. Dans les deux cas, le terme pheun est également utilisé ${ }^{10}$. On peut penser qu'au niveau du vocabulaire, l'ancien royaume du Lān Nā s'est montré un peu plus conservateur dans la mesure où, pour désigner les textes de portée historique, c'est le terme tamnān qui, dans les manuscrits, semble avoir prévalu ${ }^{11}$. Il aura là aussi fallu l'intervention d'un lettré formé à la culture siamoise pour que, à partir du début du $\mathrm{XX}^{\mathrm{e}}$ siècle, ce soit le titre Phongsāvadān Yonok qui serve à désigner l'histoire éditée et diffusée de cette région ${ }^{12}$.

La classification très judicieuse qu'opérait en $1976 \mathrm{D}$. K. Wyatt ${ }^{13}$ à propos des chroniques dispersées sur le territoire thaïlandais actuel est inadéquate dès lors que l'on parle du territoire lao. La différence, d'abord, naît du fait qu'il y avait au Siam une distinction marquée entre deux types de culture - celle du Lān Nā et celle d'Ayuthya - qui, toutes deux, avaient généré leur propre forme historiographique. Si le Lān Xāng fut forcément au contact de l'une et de l'autre, il n'en reste pas moins qu'en fonction de facteurs culturels et historiques différents, il donna naissance à des traditions annalistiques qui furent spécifiques et pour lesquelles d'autres critères de classification existent. Ces critères sont en premier lieu d'ordre géographique : le Lān Xāng fut un ensemble de muang ou petites principautés qui se signalaient par des particularismes locaux très forts. On a toujours eu tendance à oublier l'importance de ces disparités dans la mesure où, jusqu'à présent, l'histoire du Laos, telle qu'elle est connue dans ses grandes lignes, a été écrite essentiellement à partir des sources luang-prabanaises. Il est vrai qu'il s'agit là des documents les plus riches en informations, du fait de l'ancienneté de leur rédaction mais également de leur relative préservation. À côté des traditions septentrionales, il exista cependant, d'une façon certes plus tardive, des traditions historiographiques centrales et méridionales, notamment à Vientiane et à Champassak. Ces dernières, souvent délaissées, ne contribuent pas moins à donner de l'histoire du Laos une image corrigée et à l'historien une perception plus juste dans sa critique des matériaux.

9. Par convention, nous aurions pu choisir de traduire systématiquement phongsāvadān par « annales » et tamnān par «chronique ». Dans ce cas, il nous aurait cependant fallu trouver un nouveau terme pour nithān, qui désigne en principe la petite histoire alors qu'il s'applique en ce qui concerne le Nithān Khun Borom à un texte de caractère historique. Nous avons donc choisi d'employer plutôt librement les traductions « chronique » et « annales », sachant que lorsque la distinction entre les types de texte est pertinente, nous gardons les termes originaux tamnān, phongsāvadān, nithān, etc.

10. On trouvera par exemple à la Bibliothèque nationale de Vientiane le Tamnān Nakhon Vieng Chan, le Pheun Vieng Chan et le Pheun Muang Burichan (versions d'un même texte légendaire qui s'attache à la toponymie sacrée) ; le Tamnān Thāt Phanom et le Pheun Thāt Phanom ; le Pheun Muang Săy Fong ; le Tamnān Phra Bāng et le Pheun Phra Bāng. Si le Pheun Muang Luang Prabang est un phongsāvadān, il en va différemment avec le Pheun Muang Lān Xāng, qui est un texte entièrement fondé sur la légende. Proches d'un point de vue sémantique, on citera aussi les textes connus sous le nom de kong din (Kong Din Muang Luang Prabang et Kong Din Muang Phuan); ils s'attachent généralement aux limites sacrées et aux mythes de fondation.

11. Voir Lān Nā Literature: Catalogue of Palm-leaf Texts on Microfilm at the Social Research Institute, 1986.

12. Phraya Prajakicakaracakra, Ruang Phongsāvadān Yonok [Histoire du pays Yonok], 1908.

13. D. K. Wyatt (1994: 1-21) oppose les trois grandes traditions de tamnān (Tamnan of the Distant Past, Universal Histories, Monumental Tamnan) qui regroupent des textes le plus souvent très anciens et originaires du nord de l'actuelle Thaïlande à la tradition plus récente du phongsāvadān associée au royaume d'Ayuthya. 
Les chroniques luang-prabanaises véhiculent des données que l'on a pu reconnaître comme historiques dès lors qu'elles abordent le second quart du XIV ${ }^{\mathrm{e}}$ siècle, c'est-à-dire l'époque de Fā Ngum. Ces données, au départ, étaient sans doute transmises de façon orale et il est fort possible qu'elles ne commencèrent à être consignées par écrit qu'à partir du milieu du $\mathrm{XV}^{\mathrm{e}}$ siècle. On s'interroge toujours sur la date de la diffusion de l'écriture au Laos ${ }^{14}$. Ce qui apparaît en tout cas sûr, au regard des données épigraphiques, c'est qu'il existait à partir de l'extrême fin du $\mathrm{XV}^{\mathrm{e}}$ siècle des inscriptions commémoratives aussi bien à Luang Prabang que dans la région de Vientiane (Gagneux, 1977 : 35, 67). Deux écritures étaient alors employées, toutes deux issues de modèles provenant du nord de la Thaïlande ${ }^{15}$. Le $X V^{\text {e }}$ siècle fut au Lān Nā une époque florissante pour les études bouddhiques et la propagation des textes. Les relations culturelles entre les différentes régions se renforcèrent et c'est à l'évidence au travers des liens noués avec la région de Chiang Mai que se développèrent à Luang Prabang des traditions historiographiques. La lecture du colophon de certaines versions indique que la première rédaction du Nithān Khun Borom fut apparemment l'œuvre d'un bonze appelé Mahā Thep Luang Chao et qu'elle pourrait dater des dernières années du règne de Chao Vixun, un peu avant 1520 (Viravong et Uthensakda, 1967). Elle fut complétée sous le premier règne du Phrayā Sēn Surintha (autour de 1573) par un scribe nommé Phra Ariya Vangso et l'ensemble que forment ces deux parties constitue sans doute le noyau initial relativement préservé d'une chronique à laquelle on a rajouté par la suite des fragments de phongsāvadān. Les phongsāvadān, s'ils n'offrent aucun élément permettant une datation précise, ne sont certainement pas d'une rédaction postérieure à celle du Nithān Khun Borom. Sur les périodes les plus anciennes, en effet, ils apportent un éclairage beaucoup plus juste (voire plus objectif) que celui-ci. Les phongsāvadān apparaissent en fait comme un ensemble de variantes issues d'un même texte de départ. Ils tinrent véritablement le rôle d'annales dynastiques et furent alimentés, d'une façon plus ou moins conséquente suivant les époques, jusqu'à la fin du XIX $\mathrm{X}^{\mathrm{e}}$ siècle. Il reste que leur contenu est assez bref et permet à peine de suivre d'une façon linéaire l'ensemble des règnes qui se succédèrent. Étroitement liés à la terre de Luang Prabang, les phongsāvadān n'évoquent l'histoire de Vientiane, à partir de la scission du Lān Xāng au début du XVIII ${ }^{\mathrm{e}}$ siècle, que par de rares allusions relatives en général à des conflits.

Il n'est guère possible, au regard de ce qu'il nous reste des chroniques de Vientiane, d'avoir une idée précise de l'ensemble des traditions historiques qui se sont développées dans le centre du Lān Xāng. Les événements tragiques de 1828 (la destruction de Vientiane par les armées siamoises) ne se résument pas simplement à une défaite militaire lao. Ils eurent un impact plus profond encore dans la mesure où ils se soldèrent par la disparition complète d'une entité politique régionale et avec elle de la dynastie qui dirigeait sa destinée. Les insignes de cette ancienne puissance furent accaparés ou détruits. Les quelques documents qui subsistèrent nous sont connus, autant que possible, par des versions qui demeurèrent à Luang Prabang ou furent plus tard éditées en langue thaïe. Ils consistent essentiellement en des chronologies qui, par leur sécheresse et leurs données obscures, n'ont guère retenu jusqu'à présent l'attention des spécialistes ${ }^{16}$. Ces textes

14. La question est développée dans Dhawaj Poonotoke, Silachareuk Issan - The Northeastern Thai Inscriptions, s. d.

15. La première est appelée «tham » (P. dhamma : «doctrine bouddhique ») et sert à noter plus particulièrement les textes religieux. La seconde a un caractère plus laïque et civil. Ces deux écritures dérivent respectivement des modèles « tham » et « fak khām » du Lān Nā.

16. On mettra à part Pheuiphanh et Mayouri Ngaosyvathn, qui se sont particulièrement intéressés à l'histoire du royaume de Vientiane durant les décennies précédant sa disparition. 
s'avèrent pourtant essentiels dans la mesure où ils relaient à partir du milieu du XVI $\mathrm{X}^{\mathrm{e}}$ siècle la tradition septentrionale des phongsāvadān profondément affectée, semble-t-il, par le recentrage de la capitale. Ils éclairent d'un jour nouveau la succession des règnes à Vientiane depuis la mort de Setthāthirāt (1571) et viennent utilement recouper les sources épigraphiques. Ils ne laissent malheureusement rien transparaître de l'organisation socioéconomique du royaume.

Les chroniques du Sud sont surtout représentées par la tradition historiographique développée autour des princes de Champassak. Il en existe plusieurs versions, très proches les unes des autres, qui portent indifféremment le titre de tamnān ou de phongsāvadān. C. Archaimbault les donne toutes pour " des annales de rédaction récente (début du XIX ${ }^{\mathrm{e}}$ siècle) qui sont des copies ou des remaniements de textes anciens non datés » (Archaimbault, 1961 : 579). Les données qu'elles véhiculent ne deviennent historiquement utilisables qu'à partir du début du XVIII ${ }^{\mathrm{e}}$ siècle. Elles se rapprochent par leur esprit des phongsāvadān rédigés à Luang Prabang.

Il faudra sans doute mettre à part les chroniques relatant l'histoire de la région de Xieng Khuang ou Muang Phuan. D'abord parce qu'elles relèvent d'un groupe ethnique légèrement différent de celui des Lao (même si cette différence, aujourd'hui, tend de plus en plus à s'estomper), ensuite parce qu'elles présentent - ainsi que le rapporte C. Archaimbault qui en a étudié les différentes recensions - ce défaut encore plus marqué que dans les annales lao d'être de caractère composite et d'apparaître comme un "mélange incohérent de morceaux hétérogènes" (Archaimbault, 1967: 558). Les chroniques de Luang Prabang servant souvent de sources d'inspiration à ces textes, la tradition du Nithān Khun Borom se mêle ici étroitement à celle des phongsāvadān.

\section{Desseins et moyens, le rapport au sacré}

Comme on le sait, l'objet premier des annales dynastiques est de légitimer un pouvoir en place. Les chroniques du Laos n'échappent pas à cette règle même si elle se traduit parfois fort différemment suivant les textes.

Hormis les chroniques courtes de Vientiane, toutes les annales du Laos se réfèrent à un passé lointain où se distingue, d'une façon souvent fabuleuse, un ancêtre, premier maillon d'une dynastie. En cela, les chroniques laotiennes ne diffèrent guère de leurs cousines du Lān Nā, ni même de celles des royaumes voisins ${ }^{17}$. Le mythe le plus important est ici celui de Khun Borom, envoyé sur terre par le Phrayā Thēn (le roi des dieux) pour gouverner les hommes et organiser leur territoire. Le personnage a cependant une destinée assez courte: son importance découle surtout du fait qu'il donne naissance à une progéniture appelée ensuite à régner sur les pays t'ai. Le royaume de Luang Prabang désigné sous le nom du Muang Swa-occupe une position toute particulière dans la mesure où il échoit au fils aîné, Khun Lo, ce qui lui confère une certaine prééminence par rapport aux royaumes voisins tels ceux d'Ayuthya et du Lān $N a{ }^{18}$. Le fait sera particulièrement mis en valeur dans le Nithān Khun Borom car il servira de prétexte à l'arrêt des conquêtes de Fā Ngum : invincible, celui-ci ne trouve alors de limites à son entreprise que dans le respect teinté d'un sentiment de supériorité qu'il témoigne à l'encontre de ses cousins, héritiers des frères cadets de Khun Lo. Au-delà de son origine divine, l'ancêtre Khun Borom confère à ses descendants une autre légitimité dans la

17. On citera par exemple le mythe de Lavacangkaraja dans la Chronique de Chiang Mai. Voir C. Notton, 1932 : 12 ; D. K. Wyatt, A. Wichienkeeo, 1995 : 5.

18. Précisons que le mythe de Khun Borom n'apparaît pas dans les chroniques de ces deux royaumes. 
mesure où il fixe d'une façon définitive un code de conduite destiné aux souverains que la coutume perpétuera. C'est encore une fois le Nithān qui, dans sa rédaction, conserve le mieux la mémoire de ces recommandations ${ }^{19}$. Celles-ci sont associées à un rituel où la première place est réservée aux divinités célestes, lesquelles sont d'ailleurs susceptibles d'être rejointes par les âmes des monarques descendants de Khun Lo que leur illustre origine rend aptes à passer au rang de génies protecteurs (Archaimbault, $1973: 118$ ).

Si par leurs données mythiques les annales septentrionales rendent compte d'une façon particulièrement forte du caractère divin de la monarchie, il en va quelque peu différemment des traditions annalistiques du Centre et du Sud Laos. On ne s'attardera pas ici sur les quelques textes légendaires qui s'attachent aux premiers souverains de Vientiane. Il n'existe pas, en effet, de continuité entre ces règnes et ceux des souverains du Lān Xāng qui s'installèrent là à partir de la première moitié du XVI ${ }^{\mathrm{e}}$ siècle. À Champassak, le travail des historiographes fut certainement difficile dans la mesure où il s'agissait de mettre en valeur l'identité d'une dynastie dont le pouvoir ne fut effectif qu'à partir du début du XVIII ${ }^{\mathrm{e}}$ siècle. C'est la littérature et les traditions orales qui vinrent alors faciliter l'œuvre des scribes. C. Archaimbault a habilement démontré l'influence que celles-ci exercèrent dans la rédaction des chroniques et la légitimation du pouvoir en place. Tout se passe en fait comme si le Sud avait longtemps été une région autonome gouvernée par des hommes de mérites. Des changements se seraient pourtant produits au tournant du XVII et du XVIII ${ }^{\mathrm{e}}$ siècle : d'une part, la faute d'une reine jetant le discrédit sur la lignée régnante locale; les événements de Vientiane, d'autre part, qui devaient se répercuter par l'intermédiaire de transfuges sur la destinée politique de Champassak. La transmission du pouvoir se serait alors effectuée par le biais d'un bonze vertueux et adoré qui aurait installé sur le trône du Sud un prince de Vientiane, dernier rejeton légitime d'une dynastie du Nord bafouée (Archaimbault, 1961 : 531).

Toutes les annales du Nord, avant même que n'intervienne le mythe de l'ancêtre descendu du ciel, s'attachent à la configuration sacrée du territoire destiné à recevoir le premier souverain du muang Luang Prabang. Nithān et phongsāvadān commencent en effet par l'intervention de deux ermites qui placent en quatre points bien distincts les piliers de fondation de la future cité ${ }^{20}$. L'emplacement du palais ayant lui aussi été marqué, les deux personnages firent alors appel à l'ensemble des génies ophidiens et terrestres pour leur recommander d'exercer une surveillance attentive sur tout le territoire. En échange de cette protection, il fut décidé que les futurs rois de Luang Prabang offriraient aux divinités d'en bas un certain nombre de cadeaux à intervalles réguliers. Pour les chroniques, suivies en cela par tout un rituel très complexe, la légitimité du souverain se fait également par son attachement au sol et aux puissances sacrées que ce dernier recèle. Le roi est « maître de la terre » (Chao Phèn Din), il est l'intermédiaire avec les forces souterraines, aquatiques et aériennes et c'est là aussi le rôle des textes que de le rappeler ${ }^{21}$. Les traditions légendaires du Centre Laos - qu'elles mettent en scène le personnage de Burichan le Rondouillard ${ }^{22}$ ou, qu'à l'exemple des chroniques du Nord,

19. Voir Maha Sila Viravong et Noan Uthensakda, Nithān Khun Borom, Vientiane, éd. du Ministère des Cultes, 1967, p. 28-31 ; C. Archaimbault, "La naissance du monde selon les traditions lao », in Structures religieuses lao (Rites et Mythes), 1973, p. 112-113.

20. C. Archaimbault, «La naissance du monde... », op. cit., a déjà noté les différences importantes qui, tant dans le fond que dans la forme, opposaient pour les temps mythiques les deux traditions. Les phongsāvadān offrent un récit plus développé où il est fait référence, entre autres, au déluge.

21. La croyance populaire en la réincarnation du Prince Phetsarath (le dernier Vice-roi du Laos) en un Nāga procède directement de cette tradition.

22. Voir C. Archaimbault, 1980. 
elles fassent état de la reconnaissance du site de Vientiane par des ermites - ont ce même objet de créer une relation intime entre un sol a priori sacralisé et la dynastie royale qui viendra s'y installer et gouverner.

$\mathrm{Si}$, dans les textes les plus anciens, les mythes légitimants se rattachent essentiellement à des traditions animistes, il en va différemment avec des textes de rédaction plus récente. Nous connaissons un phongsāvadān de Luang Prabang (Phinit, 1987) - élaboré aux alentours de 1870 - dont l'originalité réside essentiellement dans le fait que le Bouddha intervient avant même les ancêtres fondateurs. En cela, il se rattache directement à un certain nombre de textes hagiographiques produits au Laos à des époques plus anciennes, mais également à une tradition historiographique issue du Lān $\mathrm{Na}^{23}$. Au travers de l'imagerie bouddhique, les souverains du Lān Xāng (et de Luang Prabang en particulier) trouvent ici une double légitimation. Par le sol, d'abord, dans la mesure où, devançant le travail des ermites, le Bouddha laisse sur le sol du muang les traces qui attesteront de son élection en tant que terre touchée par la grâce du dharma. D'une façon plus directe, ensuite, dans la mesure où la dynastie régnante trouve la confirmation de son rôle dans les prédictions même que faisait le Bouddha lorsqu'il parlait de la terre d'élection d'une future lignée de souverains vertueux. À un autre niveau, c'est également l'image du monarque en tant que personnage dépassant la condition humaine que véhiculent les chroniques. Il est en premier lieu celui qui possède le plus de mérites (boun) d'une façon à la fois innée et acquise : innée, car, dans la tradition bouddhique locale - particulièrement mise en valeur par la littérature -, la condition de roi s'explique par les perfections qu'il a atteintes durant ses vies antérieures ${ }^{24}$. Acquise, car, de par le prestige même de sa fonction, le roi possède avant tout autre homme la richesse qui, redistribuée d'une certaine façon (la construction de temples, de stüpa, des donations faites à la communauté religieuse), lui confère un capital supérieur de mérites. Cette image du monarque vertueux trouve sa graduation ultime dans l'idée du souverain universel (cakravartin) ${ }^{25}$. Il dépasse à ce stade le genre humain pour accéder, vivant, à un rang proche de la divinité ; il est celui qui protège et diffuse la Loi (dharma), celui à qui on doit une entière obéissance. C'est dans le Nithān Khun Borom, rédigé à une époque où le bouddhisme trouvait une nouvelle vigueur, que cette image du pouvoir légitimé par la religion transparaît le plus.

Il est intéressant de relever ici le rôle que purent tenir certaines images précieuses du Bouddha dans la légitimation même du pouvoir du souverain. Celui du Phra Bāng fut à cet égard exemplaire car pluridimensionnel et d'une certaine façon beaucoup plus subtil. Dans le Nithān Khun Borom, en particulier, la statue se trouve être au service d'une idée de la monarchie beaucoup plus qu'au service des souverains eux-mêmes. Il est nécessaire de rappeler ici son parcours : amenée du Cambodge par la mission religieuse appelée par Fā Ngum, elle décide par elle-même, une fois arrivée à Vieng Kham (non loin de Vientiane), de ne plus quitter l'endroit, réagissant ainsi à sa façon aux exactions que le monarque commençait à commettre auprès de son peuple. Peu de temps après ce désaveu, Fā Ngum fut détrôné et exilé dans un muang voisin où il devait vivre jusqu'à la fin de ses jours. Le Phra Bāng ne bougea pas pour autant, bien des entorses à la morale devant encore se

23. On pensera en particulier à un certain nombre de tamnān cités par D. K. Wyatt, 1976 [rééd. 1994].

24. La littérature lao laïque reste influencée par les modèles canoniques et présente souvent le roi comme un bodhisattva, c'est-à-dire comme un futur Bouddha. La terminologie relative à la titulature des souverains dans les documents épigraphiques met particulièrement en évidence les qualités bouddhiques exceptionnelles qu'on leur prête.

25. L'identification d'un roi ou d'un chef local au monarque cakravartin est récurrente dans les sociétés bouddhiques d'Asie du Sud-Est. Chez les T'ais, les témoignages épigraphiques indiquent qu'elle a commencé à Sukhothai à la fin du XIII ${ }^{\mathrm{e}}$ siècle. 
produire dans les premières décennies du $X^{e}$ siècle. Ce n'est qu'à partir de la fin de ce siècle, à un moment où le bouddhisme lao se voit régénéré au contact du Lān Nā, que l'image précieuse arrive enfin à Luang Prabang. Son attitude est ici doublement importante dans la mesure où, par son premier refus, elle valorise d'autant plus le choix qu'elle fait de son nouveau protecteur, en l'occurrence un proche parent de Chao Vixun (1501-1520), commanditaire probable de la chronique. Consciemment ou non, l'historiographe sert ici directement la légitimité des souverains de la fin du $X^{e}$ et du début du $\mathrm{XVI}^{\mathrm{e}}$ siècle.

\section{La réécriture de l'histoire}

$\mathrm{Si}$, par la place qu'il accorde au mythe, le Nithān Khun Borom ne diffère guère des autres chroniques, sa structure, cependant, apparaît tout à fait particulière. L'ouvrage se divise en effet en trois parts quasiment égales qui toutes ressortissent à des épopées : celle de Khun Borom et de Khun Lo, les ancêtres légendaires; celle de Fā Ngum, le roi conquérant et unificateur; et dans une certaine mesure celle de tous les souverains qui par la suite régnèrent jusqu'au début $\mathrm{du} \mathrm{XVI}^{\mathrm{e}}$ siècle. Fā Ngum, de par la position qu'il occupe, jouit d'une faveur toute particulière. Il est le noyau de l'œuvre, personnage hybride qui hérite d'une fabuleuse destinée. Il est également celui qui, en fonction même de l'œuvre qu'il accomplira, donnera sa légitimité à tous ceux de son sang qui régneront ensuite sur le Lān Xāng. Si, comme le laisse supposer un colophon, Vixun fut bien le commanditaire du premier livre du Nithān Khun Borom, il trouvait donc tout avantage à y apparaître en dernière position, comme l'ultime maillon d'une longue et précieuse chaîne, et la chronique ressemblerait ainsi à un brevet d'investiture. On a pu d'ailleurs se demander si d'une certaine façon elle ne constituait pas encore bien plus que cela : comme une sorte d'instrument légitimant a posteriori une prise du pouvoir. Lorsque l'on s'attache aux événements qui précédèrent directement ce règne et que l'on compare les données fournies par le Nithān à celles transmises par les phongsāvadān, il est en effet curieux de constater à quel point, dans le premier texte, la période est malmenée par l'historiographe. Aux illogismes chronologiques flagrants qui caractérisent les dernières années du $\mathrm{XV}^{\mathrm{e}}$ siècle s'ajoutent en effet, sur des faits essentiels qui marquèrent l'histoire du Lān Xāng à cette époque et que nombre d'autres sources rapportent (par exemple, le règne de Thēn Kham), des silences et des omissions qui ne s'expliquent que par une volonté délibérée d'occultation, voire de falsification ${ }^{26}$. On sait, au travers des données que livrent en particulier les annales chinoises, que l'accession au trône de Chao Vixun s'effectua probablement dans d'étranges conditions. Le premier livre du Nithān, dans ce cas-là, aurait pu avoir pour fonction de maquiller une usurpation et d'entériner une version officielle de l'histoire.

Les chroniques lao offrent d'autres exemples où des événements se trouvent ainsi occultés, mais il s'agit alors moins de légitimer un pouvoir que d'offrir à la postérité une image qui apparaisse comme la plus positive possible. Dans un certain nombre de phongsāvadān, s'agissant du début du XVIII ${ }^{\mathrm{e}}$ siècle, on trouve ainsi le récit largement développé des pérégrinations d'un personnage dont la destinée semble soumise à des contingences supérieures. Il s'agit de Ong Nok - également appelé Khamom Noy-, souverain éphémère de Luang Prabang avant d'être appelé à régner au Lān Nā sous le nom de Ong Kham. La comparaison entre les annales de Chiang Mai et celles de Luang

26. A. Doré (1987) donne un certain nombre d'arguments allant dans ce sens, notamment à propos du silence fait dans le Nithān sur le règne de Thēn Kham. 
Prabang s'avère ici pleine d'enseignements. En effet, si chacune d'elles fait état de l'existence du personnage, la présentation qu'elles en donnent, par contre, se révèle fondamentalement différente : à l'image édifiante et glorieuse que véhiculent les secondes s'oppose celle à la fois terne et trouble que proposent les premières. La raison en est simple même si elle n'apparaît qu'au travers d'une analyse minutieuse du canevas événementiel. La différence résulte en effet d'une confusion (volontaire ou non) faite par les historiographes lao entre deux personnages bien distincts et que leurs intérêts opposaient. Ong Nok - devenu personnage de synthèse - récupéra alors à son profit une identité et un ensemble de caractéristiques qui, dans la réalité, ne lui appartenaient pas (Lorrillard, 1995: 234). Le tout replacé dans un contexte où le mythe intervenait largement, le but des annalistes de Luang Prabang était atteint : l'exorcisation, par le biais d'une idéalisation de l'histoire, d'un certain nombre de crises affectant le territoire du Lān Xāng au début du XVIII ${ }^{\mathrm{e}}$ siècle. Les textes rédigés plus tard à l'intention de Bangkok sont nettement conçus à travers cette optique. Dans ce cas-là, pourtant, tout se passe comme s'il y avait de la part de l'historiographe lao une sorte de sursaut d'orgueil. Face à ce pouvoir extérieur thaï qui juge, on masque en effet les faiblesses et les déboires de l'histoire et l'on s'autocensure. La chose est particulièrement évidente dans le texte composé aux alentours de 1880 par le Mahā Uparāt Suvanna Phommā, qui répondait ainsi à une commande du roi du Siam (Phinit, 1987). Il est en effet évident que le dignitaire lao avait à sa disposition un grand nombre de chroniques - en particulier des phongsāvadān - et qu'il s'en inspira pour rédiger l'œuvre qu'on lui demandait ${ }^{27}$. Pourtant, des événements funestes qui, durant dix ans (autour de 1430), entachèrent l'histoire du Laos sous la responsabilité d'une princesse laotienne sanguinaire, il n'est dans ce texte tardif nullement question, alors que partout ailleurs ils étaient rapportés. L'introduction dans le même texte d'un préambule rapportant l'histoire de la venue du Bouddha sur le site de Luang Prabang se situe dans une perspective tout à fait proche : elle répond, elle aussi, à un souci de «polissage » de l'histoire dans un sens moralisateur.

Il arrive, dans certains cas, que l'histoire soit réécrite à partir de faits qui ont marqué la conscience collective. Il en est ainsi du mythe qui naquit très tôt autour du roi Setthāthirāt (1548-1571). On sait que les circonstances qui entourèrent sa mort - au cours d'une campagne dans le sud du Lān Xāng - se révèlent quelque peu douteuses. Les chroniqueurs du Nord font bien état du climat de crise qui s'ensuivit, mais on peut dire que d'une façon générale, si plaie il y eut, celle-ci se cicatrisa plutôt bien. Quelques années plus tard, cependant, les annales de Luang Prabang racontent qu'un homme habile venant du Sud se serait fait passer pour la réincarnation de Setthāthirāt et aurait entraîné, derrière lui, nombre de gens qui auraient directement menacé la sécurité de Vientiane. Les textes n'indiquent pas d'une façon claire comment la révolte se termina. Toujours est-il qu'elle aboutit à la chute et au décès du « Mahā Uparāt » qui, à ce moment-là, gouvernait le Lān Xāng. Il est intéressant de remarquer que dans un texte du Sud rédigé plus tardivement - la chronique du Muang Attopeu -, tous ces événements mentionnés d'une façon rationnelle dans la tradition septentrionale réapparaissent, mais transposés cette fois-ci dans le mythe. Les traditions littéraires - qui jouent également un rôle déterminant dans les chroniques de Champassak - tiennent ici une place essentielle car elles fournissent un cadre à l'action. Setthäthirāt se transforme ainsi en un prince aux pouvoirs magiques dont les actes miraculeux suffisent à soumettre l'ensemble des populations autochtones du Sud Laos. Du rôle en amont que la légende occupait jusque-là, puisqu'elle se bornait à justifier le

27. Une partie de ces textes échappa miraculeusement aux pillages perpétrés par les Pavillons Noirs à Luang Prabang quelques années plus tard (en 1887, l'Uparāt Suvanna Phommā devait d'ailleurs trouver la mort durant ces événements) et furent traduits par A. Pavie. 
pouvoir des souverains en remontant à des « faits » légitimants antérieurs au XIV ${ }^{\mathrm{e}}$ siècle, on passe ici à un rôle en aval dans la mesure où elle vient s'ajouter " après coup " à une histoire qui par ailleurs est déjà écrite.

\section{Le rapport au pouvoir, importance du contexte}

Les annales sont rarement écrites dans un contexte neutre. L'influence qu'exerce le pouvoir sur leur contenu se situe cependant à différents niveaux selon que ce pouvoir est proche ou éloigné dans l'espace comme dans le temps. Dans tous les cas, l'historiographe fait cependant preuve d'une certaine prudence. C'est ce sentiment qui sans aucun doute anime le compositeur de la seconde partie du Nithān Khun Borom - un certain Phra Ariya Vangso -, qui rédige aux alentours de 1572. Concluant sur le règne de Setthāthirāt, l'historiographe se risque à peine à glisser une phrase où se trouve justifiée la prise du pouvoir par le Phrayā Sēn Surintha. On devine une distance, la nécessité d'un recul, comme s'il paraissait intolérable de parler de faits totalement contemporains. Dans le cas des textes rédigés au XIX $\mathrm{X}^{\mathrm{e}}$ siècle, il s'agissait là aussi de ne pas heurter le pouvoir dominant, même si celui-ci, au demeurant, était étranger. L'allégeance au Siam se traduit ici de différentes façons. Tous les contentieux qui avaient pu exister dans le passé seront abolis ou vidés de leur substance essentielle. La chose apparaît particulièrement évidente pour les événements du XVIII ${ }^{e}$ siècle et du début du XIX ${ }^{e}$, comme par exemple la révolte de Chao Anu, souverain de Vientiane, contre son suzerain siamois en $1828^{28}$.

Les chroniques sont également les vecteurs d'une idéologie qui se développe ou qui s'affaiblit. En tant que telles, elles apparaissent comme le baromètre de la puissance du pouvoir monarchique. La structure même des textes en témoigne. On se rend compte qu'en fonction des époques, la matière est plus ou moins importante et qu'à certaines périodes riches en événements s'opposent d'autres périodes totalement occultées. Le XVI ${ }^{\mathrm{e}}$ siècle apparaît à cet égard comme le plus intéressant de toute l'histoire du Lān Xāng. Cette impression ne se fonde pas seulement sur l'image que donnent les traditions annalistiques. Les sources épigraphiques, d'une part, mais également tous les vestiges qui ressortissent aux traditions artistiques (sculpture, architecture, littérature, etc.) confirment ici le fait. Le $\mathrm{XVII}^{\mathrm{e}}$ siècle pose à ce sujet un problème. L'image que nous avons de cette époque repose en effet essentiellement sur les témoignages que nous possédons de voyageurs occidentaux. D'une façon rétrospective, ceux-ci nous font croire à une espèce d'âge d'or du Lān Xāng - ce qui ne paraît pas tout à fait évident au regard de ce que nous laissent les témoignages épigraphiques et annalistiques. Le contexte régional alors relativement calme risque de fausser un peu la vision de l'historien. La crise qui, à la fin du XVII ${ }^{e}$ siècle, toucha d'une façon définitive la grandeur du Lān Xāng trouva sans doute ses fondements dans les décennies qui précédèrent la mort du roi Suriya Vongsā (1695). Les lacunes qui affectent les chroniques lao pour tout le $\mathrm{XVII}^{\mathrm{e}}$ siècle peuvent cependant également s'expliquer d'une façon fort différente. Nous avons dit en effet que les textes qui nous étaient parvenus étaient essentiellement d'origine luang-prabanaise. Or, par un curieux phénomène, dès lors que la royauté, au milieu du $\mathrm{XVI}^{\mathrm{e}}$ siècle, quitte Luang Prabang pour s'installer à Vientiane, la tradition annalistique s'appauvrit comme si ce déracinement était nuisible à son identité et à son existence même. La chose apparaît d'autant plus significative qu'à partir du début du XVIII ${ }^{\mathrm{e}}$ siècle, au moment où des princes reviennent

28. Pour ces événements, on se reportera en particulier à l'ouvrage récent de Martin Stuart-Fox, The Lao Kingdom of Lān Xāng: Rise and Decline, 1998. 
s'installer dans la capitale du Nord, la tradition annalistique, comme une plante que l'on replace dans son terreau d'origine, retrouve une nouvelle vigueur.

Les chroniques lao révèlent des éléments tout à fait spécifiques et se différencient des traditions annalistiques développées dans les pays voisins, même dans ceux touchés par le bouddhisme du Petit Véhicule. Nous avons remarqué que s'il existait entre les annales du Laos une certaine unité de culture (elles se distinguent en cela des chroniques du territoire thaïlandais actuel), de profondes oppositions les marquaient également, tant dans le fond que dans la forme. Il est nécessaire d'examiner en premier lieu les textes de Luang Prabang - puisque ceux-ci apparaissent à l'évidence comme étant les plus anciens et les plus complets -, mais on ne peut ignorer les traditions plus méridionales qui apportent nombre de compléments d'informations. Nous avons pu vérifier que les chroniques étaient avant tout un instrument légitimant, qu'elles se fondaient sur la valeur accordée à l'idée de perpétuation d'une lignée et que, surtout, elles multipliaient les références au sacré. Le souverain trouve ici son importance dans le fait qu'il se rattache à une dynastie dont l'origine est «divine ». C'est dans le cadre des légendes de fondation que s'explique en premier lieu la genèse du pouvoir royal. Le mythe se nourrit de traditions animistes qui remontent au plus loin de la culture t'ai : un rapport intime, rappelé par les textes et les rites, s'établit alors entre le roi et l'espace sur lequel il règne et que garantissent de puissants génies. Mais les traditions bouddhiques ne sont pas absentes de cette logique. Tout en réduisant l'écart entre les textes hagiographiques et les chroniques, elles tendirent à donner du souverain une nouvelle image - celle d'un être prédestiné - et lui conférèrent en même temps cette légitimité supérieure que représente le dharma. À l'instar des actes gravés dans la pierre, les chroniques eurent alors pour rôle de rappeler les libéralités royales pour garantir la religion. Dès lors, l'historiographe lao ne s'embarrassa guère pour restituer les événements avec objectivité. Il était au service d'un pouvoir et, bien plus encore, son ouvre se voyait guidée par une certaine conception de la morale et de « ce qui devait être » en matière d'histoire.

Influencée par une culture dont le poids est aujourd'hui toujours aussi fort, l'œuvre des historiens lao modernes se rattache directement à celle des historiographes anciens dont elle fait la compilation. De ce point de vue, elle nous apporte un certain nombre de clefs qui nous permettent de mieux pénétrer les mentalités du passé. De la même façon que pour les manuscrits sur lesquels elle se fonde, elle nécessitera une sérieuse approche critique dès lors que la recherche historique la prendra en compte. 


\section{Références bibliographiques}

ARCHAIMBault, Charles

1961 «L'histoire de Campasak », Journal asiatique, 249(4), p. 519-595.

1967 «Les annales de l'ancien royaume de S'ieng Khwang », Bulletin de l'École française d'Extrême-Orient, 53 (2), p. 557-673.

1973 «La naissance du monde selon les traditions lao», dans Structures religieuses lao (Rites et Mythes), Vientiane, Vithagna.

1980 Contribution à l'étude d'un cycle de légende lau, Paris, École française d'Extrême-Orient (Publications de l'École française d'Extrême-Orient, 119).

CHAMPAKEOMANY, Maha Kham Pavatsāt Lao [Histoire Lao], Vientiane, non publié, [en lao].

COLLECTIF

1986 Lān Nā Literature : Catalogue of Palm-leaf Texts on Microfilm at the Social Research Institute, Chiang Mai, Chiang Mai University.

1990 Maha Sila Viravong, Sivit Lè Phon Ngān [Maha Sila Viravong, la vie et l'œuvre], Vientiane, Institut de Recherches en Sciences sociales, [en lao].

GAGNEUX, Pierre-Marie

1977 Recueil des inscriptions du Laos, Vientiane, Service des Monuments historiques.

DORÉ, Amphay

1987 Aux sources de la civilisation lao. Contribution ethno-historique à la connaissance de la culture louang-phrabanaise, Paris, thèse d'état.

LORRILLARD, Michel

1995 Les chroniques royales du Laos. Essai d'une chronologie des règnes des souverains lao (1316-1887), Paris, thèse de doctorat, EPHE.

NGAOSYVATHN, Pheuiphanh et Mayoury

1989 «Lao historiography and historians : case study of the war between Bangkok and the Lao in 1827 », Journal of Southeast Asian Studies, 20 (1), p. 55-69.

NotTon, Camille

1932 Annales du Siam, vol. 3 : Chronique de Xieng Mai, Paris, P. Geuthner.

PHINIT, Saveng

1987 Phongsāvadān Muang Luang Prabang (Contribution à l'histoire du royaume de Luang Prabang), Paris, École française d'Extrême-Orient (Publications de l'École française d'Extrême-Orient, 141).

PhOMVONGSA, Oukham

1966 Kwām pen mã khong Lao [Histoire du Laos], Vientiane, [en lao].

POONOTOKE, Dhawaj

s. d. Silachareuk Issan - The Northeastern Thai Inscriptions, Université Rama Khamheng, [en thai]].

Pou, Saveros

1989 Nouvelles inscriptions du Cambodge, I, Paris, École française d'ExtrêmeOrient (Textes et documents sur l'Indochine, 17).

PRAJAKICAKARACAKRa, Phraya

1908 Ruang Phongsāvadān Yonok [Histoire du pays Yonok], Bangkok, [en thai]. 
STUART-FoX, Martin

1998 The Lao Kingdom of Lān Xāng : Rise and Decline, Bangkok, White Lotus.

VIRAVONG, Maha Sila

1957 Phongsāuadān Lao [Histoire du Laos], rééd. Ministère de l'Éducation nationale du Laos, 1962 ; rééd. École Fā Ngum, 1973, [en lao].

1958 History of Laos, New York, [2 éd.], Parangon Book, 1964.

VIRAVONG, Maha Sila et UTHENSAKDA, Noan

1967 Nithān Khun Borom [L'histoire de Khun Borom], Vientiane, Ministère des Cultes, [en lao].

VONGKOT RATTANA, Chao Khamhmanh

1972 Phongsāvadān Xāt Lao [Histoire de la Nation lao], Bibliothèque nationale de Vientiane, [en lao].

WYATT, David K.

1976 "Chronicle Traditions in Thai Historiography», dans Southeast Asian History and Historiography : Essays presented to D.G.E. Hall, C. D. Cowan et O. W. Wolters éd., Ithaca, Cornell University Press ; rééd. dans Studies in Thai History, Silkworm Books, 1994.

WyATT, David K. et WiCHIENKEEO, A.

1995 The Chiang Mai Chronicle, Chiang Mai, Silkworm Books.

\begin{abstract}
Some Data related to Lao Historiography

The article begins with a general appraisal of the works of the modern Laotian historians (up to 1975) on the past of their country. The particular example of the famous scholar Maha Sila Viravong is taken. His writing of Lao history appears to be a direct continuation of the work of the Laotian historiographers from the 16th century onward.

The ancient chronicles of Laos are characterised by different regional traditions which must be distinguished and correctly identified. However, the object of all of them is to legitimize a reigning dynasty. Several means have been used that are based as much on ancient animist beliefs as on the more recent traditions of the Buddhist religion. Apart from links made with legends on the origins, flagrant examples of history being rewritten can be found in the Laotian chronicles. In certain cases, it seems that awkward events were purposely obscured. In others, the historian certainly responded to a concern for moralisation and idealisation of the facts. The chronicles perfectly reflect the context during which they were written, revealing the strengths and weaknesses of local power. Careful reading of them brings a renewed perception of the Laotian past.
\end{abstract}

